Pacific Journal of Mathematics

LINEAR IDENTITIES IN GROUP RINGS. II 


\title{
LINEAR IDENTITIES IN GROUP RINGS, II
}

\author{
D. S. PASSMAN
}

In this paper we continue our study of linear identities satisfied by group rings. An algebra $E$ is said to have a polynomial part if $E$ has an idempotent $e$ such that $e E e$ satisfies a polynomial identity. Let $K[G]$ be the group ring of $G$ over $K$ and suppose that this ring is semiprime. If $\Delta$ denotes the finite conjugate subgroup of $G$, then we show that $K[G]$ has a polynomial part if and only if $[G: \Delta]<\infty$ and $\left|\Delta^{\prime}\right|<\infty$.

The notation here and the list of references (with a few exceptions) are the same as in [I] and are therefore not repeated. Results referred to from that paper have an "I" placed before the appropriate numbers so that, for example, Theorem I. 4.2 is Theorem 4.2 of [I].

1. Twisted group rings. Let $K$ be a field and let $G$ be a (not necessarily finite) group. We let $K^{t}[G]$ denote a twisted group ring of $G$ over $K$. That is $K^{t}[G]$ is an associative $K$-algebra with basis $\{\bar{x} \mid x \in G\}$ and with multiplication defined by

$$
\bar{x} \bar{y}=\gamma(x, y) \overline{x y}, \quad \gamma(x, y) \in K-\{0\} .
$$

The associativity condition is equivalent to $\bar{x}(\bar{y} \bar{z})=(\bar{x} \bar{y}) \bar{z}$ for all $x, y, z \in G$ and this is equivalent to

$$
\gamma(x, y z) \gamma(y, z)=\gamma(x, y) \gamma(x y, z) \text {. }
$$

We call the function $\gamma: G \times G / K-\{0\}$ the factor system of $K^{t}[G]$. If $\gamma(x, y)=1$ for all $x, y \in G$ then $K^{t}[G]$ is in fact the ordinary group ring $K[G]$. In this section we briefly consider the possibility that $K^{t}[G]$ satisfies a polynomial identity.

Lemma 1.1. If $x \in G$, then in $K^{t}[G]$ we have

(i ) $1=\gamma(1,1)^{-1} \overline{1}$

(ii) $\bar{x}^{-1}=\gamma\left(x, x^{-1}\right)^{-1} \gamma(1,1)^{-1} \overline{x^{-1}}$

$$
=\gamma\left(x^{-1}, x\right)^{-1} \gamma(1,1)^{-1} \overline{x^{-1}} \text {. }
$$

Proof. Setting $y=1$ in $\left({ }^{* *}\right)$ yields $\gamma(1, z)=\gamma(x, 1)$. Thus for all $x, z \in G$ we have $\gamma(1, z)=\gamma(x, 1)=\gamma(1,1)$. Therefore by $(*), \gamma(1,1)^{-1} \overline{1}$ is the identity of $K^{t}[G]$.

Now by $\left(^{*}\right)$ and the above $\gamma\left(x^{-1}, x\right)^{-1} \gamma(1,1)^{-1} \overline{x^{-1}}$ is a left inverse 
for $\bar{x}$ and $\gamma\left(x, x^{-1}\right)^{-1} \gamma(1,1)^{-1} \overline{x^{-1}}$ is a right inverse for $\bar{x}$. Thus $\bar{x}$ is invertible and $\bar{x}^{-1}$ is equal to both of these expressions.

THEOREM 1.2. Let $K^{t}[G]$ be a twisted group ring. Suppose that $G$ has a subgroup $A$ with $[G: A]=n$ and with $K^{t}[A]$ commutative. Then $K^{t}[G]$ satisfies a polynomial identity of degree $2 n$.

Proof. Using the above lemma, we see as in the proof of Theorem I. 4.2 that $K^{t}[G] \subseteq\left(K^{t}[A]\right)_{n}$, the ring of $n \times n$ matrices over $K^{t}[A]$. The result follows from the main theorem of [2].

We now consider the reverse direction.

Lemma 1.3. Let $D$ be division algebra over a field $F$ and suppose that

$$
\operatorname{dim}_{F} D<\text { cardinality of } F .
$$

Then $D$ is algebraic over $F$. If, in addition, $F$ is algebraically closed, then $D=F$.

Proof. Let $x \in D-F$. Then for all $z \in F$ we have $1-z x \neq 0$ and hence $(1-z x)$ is invertible. Clearly all such terms $(1-z x)^{-1}$ commute. Since $\operatorname{dim}_{F} D$ is less than the cardinality of the set $\left\{(1-z x)^{-1}\right\}$ there must be a nontrivial linear dependence

$$
\sum_{1}^{n} \frac{w_{i}}{1-z_{i} x}=0
$$

with $w_{i}, z_{i} \in F, w_{i} \neq 0$ and the $z_{i}$ distinct. Multiplying the above by $\Pi\left(1-z_{i} x\right)$ yields a nontrivial polynomial satisfied by $x$. Thus $D$ is algebraic over $F$. Since $F$ is central in $D$, the second statement is clear.

Proposition 1.4. Let $A$ be an abelian group and suppose that $K^{t}[A]$ satisfies a polynomial identity of degree $n$. Then $A$ has a subgroup $Z$ with $[A: Z] \leqq(n / 2)^{2}$ and with $K^{t}[Z]$ central in $K^{t}[A]$.

Proof. Let $F$ be a field extension of $K$ which is algebraically closed and with the cardinality of $F$ bigger than that of $A$. By Lemma I. 5.3, $K^{t}[A]$ satisfies a multilinear polynomial identity of degree $n$ and hence $F^{t}[A]=F \otimes_{K} K^{t}[A]$ also satisfies this identity.

Now $F^{t}[A]$ has a unit element by Lemma 1.1 and thus $F^{t}[A]$ has an irreducible representation $\rho$. Then $\rho\left(F^{t}[A]\right)$ is a primitive $F$ algebra satisfying a polynomial identity of degree $n$ and hence by a 
theorem of Kaplansky ([14], Th. 6.3.1) $\rho\left(F^{t}[A]\right) \cong D_{m}$ where $D$ is a division algebra over $F$ and $m \leqq n / 2$. Now $F$ is algebraically closed and

$$
\operatorname{dim}_{F} D \leqq \text { cardinality of } A<\text { cardinality of } F
$$

and hence by Lemma 1.3 we have $D=F$. We choose a fixed isomorphism and write $\rho\left(F^{t}[A]\right)=F_{m}$. Let tr denote the usual trace of a matrix in $F_{m}$.

Let $Z=\left\{x \in A \mid \bar{x}\right.$ is central in $\left.K^{t}[A]\right\}$. Then by $\left({ }^{*}\right)$ and Lemma 1.1 we see that $Z$ is a subgroup of $A$ and $K^{t}[Z]$ is central in $K^{t}[A]$.

Let $x \in A-Z$. Since $\bar{x}$ is not central in $K^{t}[A]$ we conclude that for some $y \in A, \bar{x}$ and $\bar{y}$ do not commute. Since $A$ is abelian we then have by $\left({ }^{*}\right), \bar{y}^{-1} \bar{x} \bar{y}=a \bar{x}$ where $a \in K \subseteq F$ and $a \neq 1$. Thus

$$
\rho(\bar{y})^{-1} \rho(\bar{x}) \rho(\bar{y})=a_{i} \rho(\bar{x}) .
$$

Since similar matrices have the same trace we obtain

$$
\begin{aligned}
\operatorname{tr} \rho(\bar{x}) & =\operatorname{tr} \rho(\bar{y})^{-1} \rho(\bar{x}) \rho(\bar{y}) \\
& =\operatorname{tr} a \rho(\bar{x})=a \operatorname{tr} \rho(\bar{x})
\end{aligned}
$$

and hence $\operatorname{tr} \rho(\bar{x})=0$ since $a \neq 1$.

Now let $x \in Z$. Then $\bar{x}$ is also central in $F^{t}[A]$ so $\rho(\bar{x})$ is central in $F_{m}$ and hence $\rho(\bar{x})=a I$ where $a$ is a nonzero scalar and $I$ is the identity matrix. This yields $\operatorname{tr} \rho(\bar{x})=a \cdot m$. If $m$ were equal to zero in $F$ then we would have $\operatorname{tr} \rho(\bar{x})=0$ for all $x \in A$ and hence

$$
\operatorname{tr}\left(F_{m}\right)=\operatorname{tr} \rho\left(F^{t}[A]\right)=0,
$$

certainly a contradiction. Thus $m \neq 0$ in $F$.

Let $x_{1}, x_{2}, \cdots, x_{r}$ be a finite set of elements in distinct cosets of $Z$. We show that $\rho\left(\bar{x}_{1}\right), \rho\left(\bar{x}_{2}\right), \cdots, \cdots, \rho\left(\bar{x}_{r}\right)$ are linearly independent over $F$. Suppose that

$$
a_{1} \rho\left(\bar{x}_{1}\right)+a_{2 ;} \rho\left(\bar{x}_{2}\right)+\cdots+a_{r} \rho\left(\bar{x}_{r}\right)=0
$$

for $a_{i} \in F$. Multiplying the above on the left by $\rho\left(\bar{x}_{\imath}^{-1}\right)$ we obtain

$$
\left.\sum_{j=1}^{r} a_{j} \rho_{\left(x_{i}^{-1}\right.} \bar{x}_{j}\right)=0 .
$$

Now for $i \neq j, \bar{x}_{i}^{-1} \bar{x}_{j}=b \bar{x}$ for some $b \in F$ and $x \in A-Z$ and hence $\operatorname{tr} \rho\left(\bar{x}_{i}^{-1} \bar{x}_{j}\right)=0$. Thus taking traces of the above expression we obtain $a_{i} m=a_{i} \operatorname{tr} I=0$ and hence $a_{i}=0$ since $m \neq 0$ in $F$. This shows that the $\rho\left(\bar{x}_{i}\right)^{\prime}$ s are linearly independent and thus $r \leqq m^{2}$. This clearly yields $[A: Z] \leqq m^{2} \leqq(n / 2)^{2}$ and the result follows.

If $R$ is a ring, we let $R^{0}$ denote the opposite ring of $R$. Thus $R^{0}=R$ as sets and addition is the same. Moreover, for $a, b \in R^{0}$ we 
have $a \circ b=b a$.

Lemma 1.5. Let $E$ be a semiprime algebra over $K$ and suppose that $E$ satisfies a polynomial identity of degree $n$. Then $E \bigotimes_{K} E^{0}$ satisfies a polynomial identity of degree $\leqq n^{2} / 2$.

Proof. Let $P$ be a prime ideal in $E$. Then $E / P$ is a prime ring satisfying a polynomial identity of degree $n$ and hence, by a theorem of Posner ([14], Th. 7.3.2), $E / P \cong\left(F_{P}\right)_{m}$ where $F_{P}$ is a field depending on $P$ and $m=[n / 2]$. Since $E$ is semiprime it follows easily that $E \subseteq R_{m}$ where $R$ is the commutative algebra $\Pi_{P} F_{P}$. Thus clearly $E^{0} \cong R_{m}$ also and

$$
E \otimes_{K} E^{0} \cong R_{m} \otimes_{K} R_{m}=\left(R \otimes_{K} R\right)_{m^{2}} .
$$

Thus by [2], $E \otimes_{K} E^{0}$ satisfies a polynomial identity of degree $2 m^{2} \leqq n^{2} / 2$.

Lemma 1.6. Let $K^{t}[G]$ be a twisted group ring. Then $K[G]$ is $K$-isomorphic to a subalgebra of $K^{t}[G] \otimes K^{t}[G]^{0}$.

Proof. Let $x, y \in G$. In $K^{t}[G]$ we have $\overline{x y}=\gamma(x, y) \overline{x y}$ so taking inverses yields $\bar{y}^{-1} \bar{x}^{-1}=\gamma(x, y)^{-1} \overline{x y}$. Thus in $K^{t}[G]^{0}$ we have

$$
\bar{x}^{-1} \circ \bar{y}^{-1}=\gamma(x, y)^{-1} \overline{x y}^{-1} \text {. }
$$

Finally in $K^{t}[G] \otimes K^{t}[G]^{0}$ we have

$$
\begin{aligned}
\left(\bar{x} \otimes \bar{x}^{-1}\right)\left(\bar{y} \otimes \bar{y}^{-1}\right) & =(\overline{x y}) \otimes\left(\bar{x}^{-1} \circ \bar{y}^{-1}\right) \\
& =\gamma(x, y) \gamma(x, y)^{-1} \overline{x y} \otimes \overline{x y}^{-1} \\
& =\overline{x y} \otimes \overline{x y} \cdot .
\end{aligned}
$$

This shows that the $K$-linear map $K[G] \rightarrow K^{t}[G] \otimes_{K} K^{t}[G]^{0}$ defined by $x \rightarrow \bar{x} \otimes \bar{x}^{-1}$ is an injective isomorphism and the result follows.

THEOREM 1.7. There exists a finite valued function $J^{\prime \prime}$ with the following property. Let $K$ be a field and let $K^{t}[G]$ be a twisted group ring which satisfies a polynomial identity of degree n. Suppose that both $K^{t}[G]$ and $K[G]$ are semiprime. Then $G$ has a subgroup $A$ with $[G: A] \leqq J^{\prime \prime}(n)$ and with $K^{t}[A]$ commutative.

Proof. Since $K^{t}[G]$ is semiprime, Lemma 1.5 implies that

$$
K^{t}[G] \otimes_{K} K^{t}[G]^{0}
$$

satisfies a polynomial identity of degree $\leqq n^{2} / 2$. Thus by Lemma 1.6 , $K[G]$ is a semiprime group ring which satisfies a polynomial identity 
of degree $\leqq n^{2} / 2$. Hence by Theorem I. 9.4, $G$ has an abelian subgroup $B$ with $[G: B] \leqq J^{\prime}\left(n^{2} / 2\right)$. Now $K^{t}[B]$ satisfies a polynomial identity of degree $n$ so by Proposition $1.4, B$ has a subgroup $A$ with $[B: A] \leqq$ $(n / 2)^{2}$ and with $K^{t}[A]$ commutative. The result follows with $J^{\prime \prime}(n)=$ $(n / 2)^{2} J^{\prime}\left(n^{2} / 2\right)$.

Two final remarks are in order. First, the examples of $\S 4$ of [3] show that even in the case of ordinary group rings, an exact converse to Theorem 1.2 does not hold without the semiprime assumption. Second, it follows easily from Theorem 3.7 of [11] that if $K[G]$ is semiprime, then so is $K^{t}[G]$. Thus the two semiprime assumptions of Theorem 1.7 can be reduced to the single assumption that $K[G]$ is semiprime.

2. Weak polynomial identities. Let $E$ be an algebra over $K$ A generalized polynonomial over $E$ is, roughly speaking, a polynomial in the indeterminates $\zeta_{1}, \zeta_{2}, \cdots, \zeta_{n}$ in which elements of $E$ are allowed to appear both as coefficients and between the indeterminates. A precise definition appears in [13]. We say that $E$ satisfies a generalized polynomial identity if there exists a nonzero generalized polynomial $f\left(\zeta_{1}, \zeta_{2}, \cdots, \zeta_{n}\right)$ such that $f\left(\alpha_{1}, \alpha_{2}, \cdots, \alpha_{n}\right)=0$ for all $\alpha_{1}, \alpha_{2}, \cdots, \alpha_{n} \in E$. The problem here is precisely what does it mean for $f$ to be nonzero. For example, suppose the center of $E$ is bigger than $K$ and let $\alpha$ be a central element not in $K$. Then $E$ satisfies the identity $f\left(\zeta_{1}\right)=$ $\alpha \zeta_{1}-\zeta_{1} \alpha$ but surely this must be considered trivial. Again, suppose $E$ is not prime. Then we can choose nonzero $\alpha, \beta \in E$ such that $E$ satisfies the identity $f\left(\zeta_{1}\right)=\alpha \zeta_{1} \beta$ and this must also be considered trivial. We avoid these difficulties by restricting the allowable form of the polynomials.

We say that $f$ is a weak polynomial of degree $n$ if

$$
f\left(\zeta_{1}, \zeta_{2}, \cdots, \zeta_{n}\right)=\sum_{\sigma \in S_{n}} \alpha_{1, \sigma} \zeta_{\sigma(1)} \alpha_{2, \sigma} \zeta_{\sigma(2)} \cdots \alpha_{n},{ }_{\sigma} \zeta_{\sigma(n)} \alpha_{n+1, \sigma}
$$

where $\alpha_{i, \sigma} \in E$. This form is of course motivated by Lemma I. 5.3. The above $f$ is said to be nondegenerate if for some $\sigma$

$$
\alpha_{1, \sigma} E \alpha_{2, \sigma} E \cdots \alpha_{n, \sigma}^{\prime} E \alpha_{n+1, \sigma}^{\prime} \neq 0 \text {. }
$$

Otherwise $f$ is degenerate.

Lemma 2.1. Suppose $K[G]$ satisfies a nondegenerate weak polynomial identity of degree $n$. Then $K[G]$ satisfies a weak polynomial identity 


$$
f\left(\zeta_{1}, \zeta_{2}, \cdots, \zeta_{n}\right)=\sum_{\sigma \in S_{n}} \alpha_{1, \sigma} \zeta_{\sigma(1)} \alpha_{2, \sigma} \zeta_{\sigma(2)} \cdots \alpha_{n, \sigma} \zeta_{\sigma(n)} \alpha_{n+1, \sigma}
$$

with $\theta\left(\alpha_{1,1}\right) \theta\left(\alpha_{2,1}\right) \cdots \theta\left(\alpha_{n+1,1}\right) \neq 0$.

Proof. Suppose $K[G]$ satisfies $f$ with the above form. Since $f$ is nondegenerate, by reordering the $\zeta$ 's if necessary, we may assume that

$$
\alpha_{1,1} K[G] \alpha_{2,1} K[G] \cdots \alpha_{n, 1} K[G] \alpha_{n+1,1} \neq 0 .
$$

Thus there exists $x_{1}, x_{2}, \cdots, x_{n} \in G$ with

$$
\alpha_{1,1} x_{1} \alpha_{2,1} x_{2} \cdots \alpha_{n, 1} x_{n} \alpha_{n+1,1} \neq 0 \text {. }
$$

If we replace $\zeta_{i}$ in $f$ by $x_{i} \zeta_{i}$ we see clearly that $K[G]$ satisfies a weak identity $f$ with $\alpha_{1,1} \alpha_{2,1} \cdots \alpha_{n+1,1} \neq 0$.

For $i=1,2, \cdots, n+1$ write

$$
\alpha_{i, 1}=\sum \beta_{i j} y_{j}
$$

where $\beta_{i j} \in K[\Delta]$ and $\left\{y_{j}\right\}$ is a set of coset representatives for $\Delta$ in $G$. Since $\alpha_{1,1} \alpha_{2,1} \cdots \alpha_{n+1,1} \neq 0$ we conclude that

$$
\beta_{1, j_{1}} y_{j_{1}} \beta_{2, j_{2}} y_{j_{2}} \cdots \beta_{n+1, j_{n+1}} y_{j_{n+1}} \neq 0
$$

for some $j_{1}, j_{2}, \cdots, j_{n+1}$. Thus if $z_{i}=y_{j_{1}} y_{j_{2}} \cdots y_{j_{i-1}}$ with $z_{1}=1$ then

$$
\beta_{1, j_{1}}^{z_{2}^{-1}} \beta_{2, j_{2}}^{z_{2}^{-1}} \cdots \beta_{n+1, j_{n+1}}^{z_{n+1}^{-1}} \neq 0 .
$$

Now $\beta_{i, j_{i}}=\theta\left(\alpha_{i, 1} y_{j_{i}}^{-1}\right)$ so

$$
\beta_{i, j_{i}}^{z_{i}^{-1}}=\theta\left(z_{i} \alpha_{i, 1} y_{j_{i}}^{-1} z_{i}^{-1}\right)=\theta\left(z_{i} \alpha_{i, 1} z_{i+1}^{-1}\right) .
$$

It therefore follows that if we replace $\zeta_{i}$ in $f$ by $z_{i+1}^{-1} \zeta_{i} z_{i+1}$ and if, in addition, we multiply $f$ on the left by $z_{1}$ and on the right by $z_{n+2}^{-1}$, then this new weak identity obtained has the required property.

The following is a slight extension of Lemma I. 1.3.

LEMMA 2.2. Let $S=\bigcup H_{r} u_{r s}$ be a finite union of cosets of the subgroups $H_{r}$ of $G$ all of infinite index and let $H$ be a subgroup of finite index in $G$. Let $\alpha_{1}, \alpha_{2}, \cdots, \alpha_{t}, \beta_{1}, \beta_{2}, \cdots, \beta_{t} \in K[G]$ and suppose that for all $x \in H-S$ we have

$$
\alpha_{1} x \beta_{1}+\alpha_{2} x \beta_{2}+\cdots+\alpha_{t} x \beta_{t}=0 .
$$

Then $\theta\left(\alpha_{1}\right) \beta_{1}+\theta\left(\alpha_{2}\right) \beta_{2}+\cdots+\theta\left(\alpha_{t}\right) \beta_{t}=0$ and the above equation holds for all $x \in H$.

Proof. We show first that $\theta\left(\alpha_{1}\right) \beta_{1}+\theta\left(\alpha_{2}\right) \beta_{2}+\cdots+\theta\left(\alpha_{t}\right) \beta_{t}=0$. 
Suppose by way of contradiction that this is not the case and let $v \in G$ be in the support of this element of $K[G]$.

Let $W$ be the intersection of $H$ and the centralizers of all elements

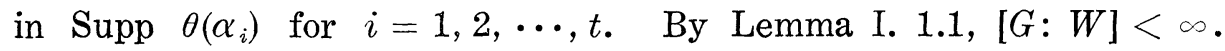
Clearly if $x \in W \cong H$ then $x$ centralizes all $\theta\left(\alpha_{i}\right)$.

Write $\alpha_{i}=\theta\left(\alpha_{i}\right)+\alpha_{i}^{\prime}$ where Supp $\alpha_{i}^{\prime} \cap \Delta=\varnothing$ and then write the finite sums

$$
\begin{aligned}
& \alpha_{i}^{\prime}=\sum a_{i j} y_{j}, \quad y_{j} \notin \Delta \\
& \beta_{i}=\sum b_{i j} z_{j} .
\end{aligned}
$$

If $y_{i}$ is conjugate to some $v z_{j}^{-1}$ in $G$ choose $g_{i j} \in G$ with $g_{i j}^{-1} y_{i} g_{i j}=v z_{j}^{-1}$.

Let $x \in W-S$. Then we must have

$$
\begin{aligned}
0= & x^{-1} \alpha_{1} x \beta_{1}+x^{-1} \alpha_{2} x \beta_{2}+\cdots+x^{-1} \alpha_{t} x \beta_{t} \\
= & {\left[\theta\left(\alpha_{1}\right) \beta_{1}+\theta\left(\alpha_{2}\right) \beta_{2} \cdots+\theta\left(\alpha_{t}\right) \beta_{t}\right] } \\
& \quad+\left[\alpha_{1}^{\prime \prime} \beta_{1}+\alpha_{2}^{\prime x} \beta_{2}+\cdots+\alpha_{t}^{\prime x} \beta_{t}\right] .
\end{aligned}
$$

Since $v$ occurs in the support of the first term it must also occur in the second and hence there exists $y_{i}, z_{j}$ with $v=y_{i}^{x} z_{j}$ or

$$
x^{-1} y_{i} x=v z_{j}^{-1}=g_{i j}^{-1} y_{i} g_{i j} .
$$

Thus $x \in \mathbf{C}_{G}\left(y_{i}\right) g_{i j}$. We have therefore shown that

$$
W \cong S \cup\left(\mathbf{U ~ C}_{G}\left(y_{i}\right) g_{i j}\right)=\left(\mathbf{U} H_{r} u_{r s}\right) \cup\left(\mathbf{U ~ C}_{G}\left(y_{i}\right) g_{i j}\right) .
$$

Since $[G: W]<\infty$ this implies that $G$ is a finite union of cosets of the subgroups $H_{r}$ and $\mathbf{C}_{G}\left(y_{i}\right)$ all of infinite index in $G$. This contradicts Lemma I. 1.2 and the first result follows.

Now write $\alpha_{i}=\Sigma y_{j} \alpha_{i j}$ where $\alpha_{i j} \in K[\Delta]$ and the $y_{j}$ are coset representatives for $\Delta$ in $G$. Then $\alpha_{i j}=\theta\left(y_{j}^{-1} \alpha_{i}\right)$ so $\alpha_{i}=\Sigma y_{j} \theta\left(y_{j}^{-1} \alpha_{i}\right)$. Since

$$
y_{j}^{-1} \alpha_{1} x \beta_{1}+y_{j}^{-1} \alpha_{2} x \beta_{2}+\cdots+y_{j}^{-1} \alpha_{t} x \beta_{t}=0
$$

for all $x \in H-S$ we conclude from the above that

$$
\theta\left(y_{j}^{-1} \alpha_{1}\right) \beta_{1}+\theta\left(y_{j}^{-1} \alpha_{2}\right) \beta_{2}+\cdots+\theta\left(y_{j}^{-1} \alpha_{t}\right) \beta_{t}=0 .
$$

Thus clearly $\alpha_{1} \beta_{1}+\alpha_{2} \beta_{2}+\cdots+\alpha_{t} \beta_{t}=0$.

Finally, let $y \in H$. Then for all $x \in H-S y^{-1}$ we have

$$
\alpha_{1} x\left(y \beta_{1}\right)+\alpha_{2} x\left(y \beta_{2}\right)+\cdots+\alpha_{t} x\left(y \beta_{t}\right)=0 .
$$

Since $S y^{-1}$ has the same form as $S$ we conclude from the preceding paragraph that

$$
\alpha_{1} y \beta_{1}+\alpha_{2} y \beta_{2}+\cdots+\alpha_{t} y \beta_{t}=0
$$


Since $y \in H$ was arbitrary, the result follows.

As an amusing corollary of this lemma we have

THEOREM 2.3. Suppose that $K$ is algebraically closed and nondenumerable and suppose that $K[G]$ is semiprime. Then $K[G]$ has a minimal right ideal if and only if $G$ is finite.

Proof. Certainly if $G$ is finite then $K[G]$ has a minimal right ideal. We now consider the converse.

Suppose $I$ is a minimal right ideal in $K[G]$. If $I^{2}=0$ then $K[G] I$ is a nonzero ideal with square zero and this contradicts Lemma I. 3.1 and the fact that $K[G]$ is semiprime. Thus $I^{2} \neq 0$ and it follows easily that $I=e K[G]$ for some idempotent $e$. By Schur's lemma, $D=e K[G] e$ is a division algebra over $K$.

If $G$ is not finite let $H$ be a countably infinite subgroup of $G$ with $H \supseteqq \operatorname{Supp} e$. We show now that $e K[H] e$ is also a division algebra. Let $\alpha \in K[H]$ and suppose that $e x e \neq 0$. Then there exists $\beta \in K[G]$ with $(e \alpha e)(e \beta e)=e$. Write $\beta=\beta_{0}+\beta_{1}$ where $\beta_{0} \in K[H]$ and Supp $\beta_{1} \cap H=\varnothing$. Then

$$
(e \alpha e)\left(e \beta_{0} e\right)+(e \alpha e)\left(e \beta_{1} e\right)=e .
$$

Since $e \in K[H]$ we have Supp $(e \alpha e)\left(e \beta_{1} e\right) \cap H=\varnothing$ and thus

$$
(e \alpha e)\left(e \beta_{0} e\right)=e .
$$

Now $e K[H] e$ is a division algebra of countably dimension over $K$. Since $K$ is algebraically closed and nondenumerable we have $e K[H] e=$ $K e$ by Lemma 1.3. Thus for all $x \in H$ we have $e x e=a_{x} e$ for some $a_{x} \in K$. Now clearly there are only finitely many $x \in H$ say $x_{1}, x_{2}, \cdots$, $x_{t}$ with $\operatorname{Supp}(e x e) \cap \operatorname{Supp} e \neq \varnothing$. Thus $e x e=0$ for all $x \in H-\bigcup\left\{x_{i}\right\}$. We think of the $\left\{x_{i}\right\}$ as cosets of $\langle 1\rangle$. Since $[H:\langle 1\rangle]=\infty$ we conclude by Lemma 2.2 applied to the group ring $K[H]$ that exe $=0$ for all $x \in H$. Thus $e^{2}=0$, a contradiction and the result follows.

3. Reduction to $\Delta$. In this section we prove the following result.

Theorem 3.1. Suppose that $K[G]$ satisfies a nondegenerate weak polynomial identity of degree $n$. Then $[G: \Delta]<\infty$ and $K[\Delta]$ satisfies a nondegenerate weak polynomial identity of degree $n$.

Proof. We show first that $[G: \Delta]<\infty$ following the proof of Theorem I. 6.2. Assume by way of contradiction that $[G: \Delta]=\infty$. By Lemma 2.1 we can assume that $K[G]$ satisfies the weak identity 


$$
f\left(\zeta_{1}, \zeta_{2}, \cdots, \zeta_{n}\right)=\sum_{\sigma \in S_{n}} \alpha_{1, \sigma} \zeta_{\sigma(1)} \alpha_{2, \sigma} \zeta_{\sigma(2)} \cdots \alpha_{n, \sigma} \zeta_{\sigma(n)} \alpha_{n+1, \sigma}
$$

with $\theta\left(\alpha_{1,1}\right) \theta\left(\alpha_{2,1}\right) \cdots \theta\left(\alpha_{n+1,1}\right) \neq 0$.

For $j=1,2, \cdots, n+1$ define $T_{j} \subseteq S_{n}$ by

$$
T_{j}=\left\{\sigma \in S_{n} \mid \sigma(1)=1, \sigma(2)=2, \cdots \sigma(j-1)=j-1\right\} .
$$

Then $T_{1}=S_{n}, T_{n+1}=\{1\}$ and $T_{j}$ is just an imbedding of $S_{n+1-j}$ in $S_{n}$. Define the weak polynomials $f_{j}$ of degree $n+1-j$ by

$$
\begin{aligned}
& f_{j}\left(\zeta_{j}, \zeta_{j+1}, \zeta_{n}\right) \\
= & \sum_{\sigma \in T_{j}} \theta\left(\alpha_{1, \sigma}\right) \theta\left(\alpha_{2, \sigma}\right) \cdots \theta\left(\alpha_{j-1, \sigma}\right) \alpha_{j, \sigma} \zeta_{\sigma(j)} \alpha_{j+1, \sigma} \zeta_{\sigma(j+1)} \cdots \alpha_{n, \sigma} \zeta_{\sigma(n)} \alpha_{n+1, \sigma} .
\end{aligned}
$$

Thus $f_{1}=f$ and

$$
f_{n+1}=\theta\left(\alpha_{1,1}\right) \theta\left(\alpha_{2,1}\right) \cdots \theta\left(\alpha_{n, 1}\right) \alpha_{n+1,1}
$$

is a nonzero constant function since

$$
\theta\left(\alpha_{1,1}\right) \theta\left(\alpha_{2,1}\right) \cdots \theta\left(\alpha_{n+1,1}\right) \neq 0 .
$$

Let $\mathscr{C}$ be the set of monomial polynomials obtained as follows. For each $\sigma$ we start with

$$
\alpha_{1, \sigma} \zeta_{\sigma(1)} \alpha_{2, \sigma} \zeta_{\sigma(2)} \cdots \alpha_{n, \sigma} \zeta_{\sigma(n)} \alpha_{n+1, \sigma}
$$

and we modify it by (1) deleting some but not all of the $\zeta_{i}$; (2) replacing some of the $\alpha_{i, \sigma}$ by $\theta\left(\alpha_{i, \sigma}\right)$; and (3) replacing some of the $\alpha_{i, \sigma}$ by 1 . Then $\mathscr{H C}$ consists of all such monomials obtained for all $\sigma \in S_{n}$ and clearly $\mathscr{C}$ is a finite set. Note that $\mathscr{C}$ may contain the zero monomial but it contains no nonzero constant monomial since in (1) we do not allow all the $\zeta_{i}$ to be deleted.

For $j=1,2, \cdots, n+1$ define $\mathscr{M}_{j} \subseteq \mathbb{l}$ by $\mu \in \mathscr{C}_{j}$ if and only if $\zeta_{1}, \zeta_{2}, \cdots, \zeta_{j-1}$ do not occur as variables in $\mu$. Thus $\mathscr{C}_{1}=\mathscr{l}$ and $\mathscr{A}_{n+1} \subseteq\{0\}$ where 0 is the zero monomial.

Under the assumption that $[G: \Delta]=\infty$ we prove by induction on $j=1,2, \cdots, n+1$ that for all $x_{j}, x_{j+1}, \cdots, x_{n} \in G$ either

$$
f_{\jmath}\left(x_{j}, x_{j+1}, \cdots, x_{n}\right)=0
$$

or there exists $\mu \in \mathscr{H}_{j}$ with Supp $\mu\left(x_{j}, x_{j+1}, \cdots, x_{n}\right) \cap \Delta \neq \varnothing$. Since $f=f_{1}$ is an identity satisfied by $K[G]$, the result for $j=1$ is clear.

Suppose the inductive result holds for some $j<n+1$. Fix $x_{j+1}, x_{j+2}, \cdots x_{n} \in G$ and let $x \in G$ play the role of the $j^{t h}$ variable. Let $\mu \in \mathscr{C}_{j+1}$. If $\operatorname{Supp} \mu\left(x_{j+1}, \cdots, x_{n}\right) \cap \Delta \neq \varnothing$ we are done. Thus we may assume that $\operatorname{Supp} \mu\left(x_{j+1}, \cdots, x x_{n}\right) \cap \Delta=\varnothing$ for all $\mu \in \mathbb{l}_{j+1}$. Set $\mathscr{N}_{j}-\mathscr{N}_{j+1}=\mathscr{N}_{j}$. 
Now let $\mu \in \mathscr{N}_{j}$ so that $\mu$ involves the variable $\zeta_{j}$. Write $\mu=\mu^{\prime} \zeta_{j} \mu^{\prime \prime}$ where $\mu^{\prime}$ and $\mu^{\prime \prime}$ are monomials in the variables $\zeta_{j+1}, \cdots, \zeta_{n}$. Then Supp $\mu\left(x, x_{j+1}, \cdots, x_{n}\right) \cap \Delta \neq \varnothing$ implies that

$$
x \in h^{\prime-1} \Delta h^{\prime-1}=\Delta h^{\prime-1} h^{\prime-1}
$$

where $h^{\prime} \in \operatorname{Supp} \mu^{\prime}\left(x_{j+1}, \cdots, x_{n}\right)$ and $h^{\prime \prime} \in \operatorname{Supp} \mu^{\prime \prime}\left(x_{j+1}, \cdots, x_{n}\right)$. Thus it follows that for all $x \in G-S$ where

$$
S=\bigcup_{\substack{\mu \in-r_{j} \\ h^{\prime}, h^{\prime \prime}}} \Delta h^{\prime-1} h^{\prime \prime-1}
$$

we have $\operatorname{Supp} \mu\left(x, x_{j+1}, \cdots x_{n}\right) \cap \Delta=\varnothing$ for all $\mu \in \mathscr{A}_{j}$. Thus by the inductive result for $j$ we conclude that for all $x \in G-S$ we have $f_{j}\left(x, x_{j+1}, \cdots, x_{n}\right)=0$. Note that $S$ is a finite union of cosets of $\Delta$, a subgroup of infinite index in $G$.

Now clearly

$$
\begin{aligned}
& f_{j}\left(x, x_{j+1}, \cdots, x_{n}\right) \\
= & \sum_{\sigma \in T_{j+1}} \theta\left(\alpha_{1, \sigma}\right) \theta\left(\alpha_{2, \sigma}\right) \cdots \theta\left(\alpha_{j-1, o}\right) \alpha_{j, o} x \alpha_{j+1, o} x_{\sigma(j+1)} \cdots x_{\sigma(n)} \alpha_{n+1, o} \\
+ & \sum_{\mu \in \mathscr{M}_{j+1}} \mu\left(x_{j+1}, \cdots, x_{n}\right) x \eta\left(x_{j+1}, \cdots, x_{n}\right)
\end{aligned}
$$

where the $\eta\left(\zeta_{j+1}, \cdots, \zeta_{n}\right)$ are suitable monomials. Note that

$$
\theta_{\mathrm{i}}\left[\theta\left(\alpha_{1, \sigma}\right) \theta\left(\alpha_{2, \sigma}\right) \cdots \theta\left(\alpha_{j-1, \sigma}\right) \alpha_{j, \sigma}\right]=\theta\left(\alpha_{1, \sigma}\right) \theta\left(\alpha_{2, \sigma}\right) \cdots \theta\left(\alpha_{j, \sigma}\right)
$$

and that $\theta\left(\mu\left(x_{j+1}, \cdots, x_{n}\right)\right)=0$ since by assumption

$$
\operatorname{Supp} \mu\left(x_{j+1}, \cdots, x_{n}\right) \cap \Delta=\varnothing \text {. }
$$

Now $f_{j}\left(x, x_{j+1}, \cdots, x_{n}\right)=0$ for all $x \in G-S$ and hence by Lemma 2.2 and the above few remarks we obtain

$$
\begin{aligned}
0 & =\sum_{\sigma \in T_{j+1}} \theta\left(\alpha_{1, \sigma}\right) \theta\left(\alpha_{2, \sigma}\right) \cdots \theta\left(\alpha_{j, \sigma}\right) \alpha_{j+1, \sigma} x_{\sigma(j+1)} \cdots x_{\sigma(n)} \alpha_{n+1, \sigma} \\
& =f_{j+1}\left(x_{j+1}, \cdots, x_{n}\right)
\end{aligned}
$$

and the induction step is proved.

In particular, we conclude for $j=n+1$ that either $f_{n+1} \doteq 0$ or there exists $\mu \in \mathscr{A}_{n+1}$ with $\operatorname{Supp} \mu \cap \Delta \neq \varnothing$. However, $f_{n+1}$ is known to be a nonzero constant function and $\mathscr{N}_{n+1} \subseteq\{0\}$. Hence we have a contradiction and we must therefore have $[G: \Delta]<\infty$.

We show next that for any weak identity $f$ we have

$$
0=\sum_{\sigma} \theta\left(\alpha_{1, \sigma}\right) \theta\left(\alpha_{2, \sigma}\right) \cdots \theta\left(\alpha_{n+1, \sigma}\right) .
$$

To do this it is convenient to multiply $f$ on the right by a new variable 
$\zeta_{n+1}$ so that if $S_{n} \subseteq S_{n+1}$ is the set of all permutations fixing the point $n+1$ then

$$
\sum_{\sigma \in S_{n}} \alpha_{1, \sigma} \zeta_{\sigma(1)} \alpha_{2, \sigma} \zeta_{\sigma(2)} \cdots \zeta_{\sigma(n)} \alpha_{n+1, \sigma} \zeta_{\sigma(n+1)}
$$

is a weak identity satisfied by $K[G]$.

Let $\left\{y_{j}\right\}$ be a finite subset of $G$ such that $\operatorname{Supp} \alpha_{i, \sigma} \subseteq\left\{y_{j}\right\}$ for all $i, \sigma$. Let $x_{1}, x_{2}, \cdots, x_{n+1}$ be arbitrary elements of $\Delta$. If we write each $\alpha_{i, \sigma}$ in terms of the $y^{\prime}$ s and if we put $\zeta_{i}=x_{i}$ in the above then we obtain

$$
0=\sum_{\sigma, \tau} a_{\sigma, \tau} y_{\tau_{1}} x_{\sigma(1)} y_{\tau_{2}} x_{\sigma(2)} \cdots y_{\tau_{n+1}} x_{\sigma(n+1)}
$$

where $\tau=\left(\tau_{1}, \tau_{2}, \cdots, \tau_{n+1}\right)$ runs through all $n+1$-tuples with allowable entries and $a_{\sigma, \tau} \in K$.

Fix $x_{2}, \cdots, x_{n+1} \in \Delta$. Then the above linear identity holds for all $x_{1} \in \Delta$ and $[G: \Delta]<\infty$. Thus Lemma 2.2 applies with $H=\Delta$. Let us consider the effect of this lemma on a typical term of the above. Now

$$
a_{\sigma, \tau} y_{\tau_{1}} x_{\sigma(1)} y_{\tau_{2}} x_{\sigma(2)} \cdots x_{1} \cdots y_{\tau_{n+1}} x_{\sigma(n+1)}
$$

becomes

$$
a_{\sigma, \tau} \theta\left(y_{\tau_{1}} x_{\sigma(1)} y_{\tau_{2}} x_{\sigma(2)} \cdots\right) \widehat{x}_{1} \cdots y_{\tau_{n+1}} x_{\sigma(n+1)}
$$

where $\widehat{x}_{3}$ indicates that $x_{1}$ is deleted. Since $z=y_{\tau_{1}} x_{\sigma(1)} y_{\tau_{2}} x_{\sigma(2)} \cdots \in G$ it follows that

$$
\theta(z)= \begin{cases}z & \text { if } z \in \Delta \\ 0 & \text { if } z \notin \Delta\end{cases}
$$

Now $\Delta$ is normal in $G$ and $x_{2}, x_{3}, \cdots, x_{n+1} \in \Delta$. Thus

$$
z=y_{\tau_{1}} x_{\sigma(1)} y_{\tau_{2}} x_{\sigma(2)} \cdots \in \Delta
$$

if and only if $y_{\tau_{1}} y_{\tau_{2}} \cdots \in \Delta$. Therefore by applying Lemma 2.2 we obtain setting $x_{1}=1$

$$
0=\sum_{\substack{\sigma, \tau \\ y_{\tau_{1}} y_{\tau_{2}} \cdots y_{\tau_{\sigma}-1(1)} \in \Delta}} a_{\sigma, \tau} y_{\tau_{1}} x_{\sigma(1)} y_{\tau_{2}} x_{\sigma(2)} \cdots y_{\tau_{n+1}} x_{\sigma(n+1)} .
$$

This identity holds for all $x_{2}, \cdots, x_{n+1} \in \Delta$ so we apply Lemma 2.2 successively. At the last step, since all $x_{i}$ will be 1 , we will have

$$
0=\sum_{\sigma, \tau}^{\prime} a_{\sigma, \tau} y_{\tau_{1}} y_{\tau_{2}} \cdots y_{\tau_{n+1}}
$$

where the sum is over all those $\sigma, \tau$ terms with 


$$
\begin{gathered}
y_{\tau_{1}} y_{\tau_{2}} \cdots y_{\tau_{\sigma^{-1}}(1)} \in \Delta \\
y_{\tau_{1}} y_{\tau_{2}} \cdots y_{\tau_{\sigma^{-1}(2)}} \in \Delta \\
\vdots \\
y_{\tau_{1}} y_{\tau_{2}} \cdots y_{\tau_{\sigma^{-1}}(n+1)} \in \Delta .
\end{gathered}
$$

Since $\sigma$ is a permutation the above is equivalent to

$$
y_{\tau_{1}} \in \Delta, y_{\tau_{1}} y_{\tau_{2}} \in \Delta, \cdots, y_{\tau_{1}} y_{\tau_{2}} \cdots y_{\tau_{n+1}} \in \Delta
$$

and hence equivalent to $y_{\tau_{1}}, y_{\tau_{2}}, \cdots, y_{\tau_{n+1}} \in \Delta$. Thus clearly

$$
\sum_{\sigma, \tau}^{\prime} a_{\sigma,-} y_{\tau_{1}} y_{\tau_{2}} \cdots y_{\tau_{n}}=\sum_{\sigma \in S_{n}} \theta\left(\alpha_{1, \sigma}\right) \theta\left(\alpha_{2, \sigma}\right) \cdots \theta\left(\alpha_{n+1, \sigma}\right)
$$

and the latter sum is therefore equal to zero.

Now fix $x_{1}, x_{2}, \cdots, x_{n} \in \Delta$ and-replace $\zeta_{i}$ in $f$ by $x_{i} \zeta_{i}$. This is a new weak identity satisfied by $K[G]$ and applying the above result to it we obtain

$$
0=\sum_{\sigma \in S_{n}} \theta\left(\alpha_{1, \sigma} x_{\sigma(1)}\right) \theta\left(\alpha_{2, \sigma} x_{\sigma(2)}\right) \cdots \theta\left(\alpha_{n, \sigma} x_{\sigma(n)}\right) \theta\left(\alpha_{n+1, \sigma}\right) .
$$

Since $x_{i} \in \Delta$ we have $\theta\left(\alpha_{i, \sigma} x_{\sigma(i)}\right)=\theta\left(\alpha_{i, \sigma}\right) x_{\sigma(i)}$ and hence

$$
0=\sum_{\sigma \in S_{n}} \theta\left(\alpha_{1, \sigma}\right) x_{\sigma(1)} \theta\left(\alpha_{2, \sigma}\right) x_{\sigma(2)} \cdots \theta\left(\alpha_{n, \sigma}\right) x_{\sigma(n)} \theta\left(\alpha_{n+1, \sigma}\right) .
$$

This shows immediately that $K[\Delta]$ satisfies the weak polynomial identity

$$
g\left(\zeta_{1}, \cdots, \zeta_{n}\right)=\sum_{\sigma \in S_{n}} \theta\left(\alpha_{1, \sigma}\right) \zeta_{\sigma(1)} \theta\left(\alpha_{2, \sigma}\right) \zeta_{\sigma(2)} \cdots \theta\left(\alpha_{n, \sigma}\right) \zeta_{\sigma(n)} \theta\left(\alpha_{n+1, \sigma}\right) .
$$

Moreover, $g$ is nondegenerate since

$$
\theta\left(\alpha_{1,1}\right) \theta\left(\alpha_{2,1}\right) \cdots \theta\left(\alpha_{n+1,1}\right) \neq 0
$$

and the result follows.

4. Polynomial parts. Let $E$ be an algebra over $K$. We say that $E$ has a polynomial part if and only if $E$ has an idempotent $e$ such that $e E e$ satisfies a polynomial identity.

THEOREM 4.1. Let $K[G]$ be a semiprime group ring. The following are equivalent:

(i) [G: $\Delta]<\infty$ and $\left|\Delta^{\prime}\right|<\infty$;

(ii) $K[G]$ has a polynomial part;

(iii) $K[G]$ satisfies a nondegenerate weak polynomial identity.

Proof. (i) $\Rightarrow($ ii). Since $K[G]$ is semiprime it follows from 
Theorem I. 3.6 that $\left|\Delta^{\prime}\right|$ is not zero in $K$. Set

$$
e=\frac{1}{\left|\Delta^{\prime}\right|} \sum_{x \in \Delta^{\prime}} x \in K[G]
$$

It follows easily that $e$ is a central idempotent in $K[G]$ since $\Delta^{\prime}$ is normal in $G$. Moreover, it is also easy to show that $e K[G] \simeq K\left[G / \Delta^{\prime}\right]$. Now $G / \Delta^{\prime}$ has an abelian subgroup $\Delta / \Delta^{\prime}$ of finite index. Hence by Theorem I. $4.2 K\left[G / \Delta^{\prime}\right]$ satisfies a polynomial identity and thus $K[G]$ has a polynomial part

(ii) $\Rightarrow$ (iii). Let $e$ be an idempotent such that $E=e K[G] e$ satisfies a polynomial identity. By Lemma I. 5.3 $E$ satisfies an identity of the form

$$
g\left(\zeta_{1}, \zeta_{2}, \cdots, \zeta_{n}\right)=\sum_{\sigma} a_{o} \zeta_{\sigma(1)} \zeta_{\sigma(2)} \cdots \zeta_{\sigma(n)} .
$$

If $\alpha \in K[G]$ then of course exe $E$. This shows immediately that $K[G]$ satisfies the weak polynomial identity

$$
f\left(\zeta_{1}, \zeta_{2}, \cdots, \zeta_{n}\right)=\sum_{\sigma} a_{\sigma} e \zeta_{\sigma(1)} e \zeta_{\sigma(2)} e \cdots e \zeta_{\sigma(n)} e .
$$

Moreover, $f$ is nondegenerate since $a_{\sigma} \neq 0$ for some $\sigma$ and then $a_{\sigma} e \cdot e \cdot \cdots e=a_{\sigma} e \neq 0$.

(iii) $\Rightarrow$ (i ). By Theorem 3.1, [G: $\Delta]<\infty$ and $K[\Delta]$ satisfies a nondegenerate weak polynomial identity

$$
f\left(\zeta_{1}, \cdots, \zeta_{n}\right)=\sum_{\sigma} \alpha_{1, \sigma} \zeta_{\sigma(1)} \alpha_{2, \sigma} \zeta_{\sigma(1)} \alpha_{2, o} \zeta_{o(2)} \cdots \alpha_{n, \sigma} \zeta_{o(n)} \alpha_{n+1, \sigma} \cdot
$$

By Lemma 2.1 applied to $\Delta$ we may assume that

$$
\alpha_{1,1} \alpha_{2,1} \cdots \alpha_{n, 1} \alpha_{n+1,1} \neq 0 \text {. }
$$

Let $C$ be the finitely generated subgroup of $\Delta$, generated by $\operatorname{Supp} \alpha_{i, \sigma}$ for all $i, \sigma$. Let $H$ be the intersection of the centralizers in $\Delta$ of all the elements in $\operatorname{Supp} \alpha_{i, \sigma}$ for all $i, \sigma$. By Lemma I. 1.1, $[\Delta: H]<\infty$. Clearly $K[H]$ centralizes $K[C]$. Thus if we restrict the variables of $f$ to values in $K[H]$ we see that $K[H]$ satisfies the identity

$$
g\left(\zeta_{1}, \zeta_{2}, \cdots, \zeta_{n}\right)=\sum_{\sigma} \beta_{\sigma} \zeta_{\sigma(1)} \zeta_{\sigma(2)} \cdots \zeta_{\sigma(n)}
$$

where $\beta_{\sigma}=\alpha_{1, \sigma} \alpha_{2, \sigma} \cdots \alpha_{n+1, \sigma} \in K[C]$ and $\beta_{1} \neq 0 . \quad$ By multiplying all $\beta_{\sigma}$ on the left by some $x \in C$ if necessary we may assume that $1 \in \operatorname{Supp} \beta_{1}$. Write $\beta_{\sigma}=\beta_{\sigma}^{\prime}+\beta_{\sigma}^{\prime \prime}$ where Supp $\beta_{\sigma}^{\prime} \subseteq H^{\prime} \cap C$ and

$$
\operatorname{Supp} \beta_{\sigma}^{\prime \prime} \cap\left(H^{\prime} \cap C\right)=\varnothing \text {. }
$$

Then by assumption $\beta_{1}^{\prime} \neq 0$ and we claim that 


$$
g^{\prime}\left(\zeta_{1}, \zeta_{2}, \cdots, \zeta_{n}\right)=\sum_{\sigma} \beta_{\sigma}^{\prime} \zeta_{\sigma(1)} \zeta_{\sigma(2)} \cdots \zeta_{\sigma(n)}
$$

is an identity satisfied by $K[H]$. Since $g^{\prime}$ is multilinear it suffices to check this fact by evaluating $g^{\prime}$ on elements of $H$.

Let $x_{1}, x_{2}, \cdots, x_{n} \in H$ and set $z=x_{1} x_{2} \cdots x_{n}$. Then since

$$
x_{\sigma(1)} x_{\sigma(2)} \cdots x_{\sigma(n)} \equiv x_{1} x_{2} \cdots x_{n} \bmod H^{\prime}
$$

we have

$$
x_{\sigma(1)} x_{\sigma(2)} \cdots x_{\sigma(n)}=h_{\sigma} z
$$

where $h_{\sigma} \in H^{\prime}$. Since $K[H]$ satisfies the identity $g$ we therefore have

$$
0=\left(\Sigma_{\sigma} \beta_{\sigma}^{\prime} h_{\sigma}\right) z+\left(\Sigma_{\sigma} \beta_{\sigma}^{\prime \prime} h_{\sigma}\right) z .
$$

Now $\operatorname{Supp} \beta_{\sigma}^{\prime} h_{\sigma} \subseteq H^{\prime}$ and $\left(\operatorname{Supp} \beta_{\sigma}^{\prime \prime} h_{\sigma}\right) \cap H^{\prime}=\varnothing$. This shows that

$$
0=\left(\Sigma_{\sigma} \beta_{\sigma}^{\prime} h_{\sigma}\right) z=g^{\prime}\left(x_{1}, x_{2}, \cdots, x_{n}\right) \text {. }
$$

Let $Z$ be the subgroup of $\Delta$ generated by the $\operatorname{Supp} \beta_{\sigma}^{\prime}$ for all $\sigma$. Then $Z$ is a finitely generated subgroup of $H^{\prime} \cap C$. Since $H$ centralizes $C$ it follows that $Z$ is central in $H$. Since $Z$ is finitely generated it follows that there is a finitely generated subgroup $H_{1} \subseteq H$ with $Z \subseteq H_{1}^{\prime}$. By Lemma I. 2.2 $H_{1}^{\prime}$ is finite and hence $Z$ is a finite central subgroup of $H$.

Let $F$ be the algebraic closure of $K$. Then certainly $F[H]$ also satisfies the identity $g^{\prime}$. Since $K[G]$ is semiprime and $Z \cong \Delta$, Theorem I. 3.6 implies that $|Z| \neq 0$ in $F$. Thus $F[Z]$ is a finite dimensional commutative semisimple algebra over an algebraically closed field and since $\beta_{1}^{\prime} \neq 0$ there exists a primitive idempotent $e \in F[Z]$ with $e \beta_{1}^{\prime} \neq 0$. Since $e F[Z]=e F$ we have $e \beta_{\sigma}^{\prime}=e a_{\sigma}$ for some $a_{\sigma} \in F$. Thus since $e$ is central in $F[H]$, the algebra $e F[H]$ satisfies the nontrivial ordinary polynomial identity

$$
h\left(\zeta_{1}, \zeta_{2}, \cdots, \zeta_{n}\right)=\Sigma_{\sigma} a_{\sigma} \zeta_{\sigma(1)} \zeta_{\sigma(2)} \cdots \zeta_{\sigma(n)} .
$$

Since $K[G]$ is semiprime and $H \subseteq \Delta$ it follows easily from Theorem I. 3.3 and Theorem I. 3.6 that $F[H]$ is semiprime. Since $|Z|$ is finite there results also show that $F[H / Z]$ is semiprime. In addition $F[H]=e F[H]+(1-e) F[H]$ is a ring direct sum and thus Lemma I. 3.1 implies easily that $e F[H]$ is semiprime.

We now observe that $e F[H] \cong F^{t}[H / Z]$ where the latter is some twisted group algebra of $H / Z$. This follows since

$$
e \in F[Z], e F[Z]=e F
$$

and $e$ is a central idempotent in $F[H]$. In view of the above, Theorem 
1.7 applies and we conclude that $H / Z$ has an abelian subgroup $A / Z$ with $[H / Z: A / Z]<\infty$. Since $[\Delta: H]<\infty$ we have $[\Delta: A]<\infty$. Moreover if $B$ is the intersection of the finitely many conjugates of $A$ in $\Delta$ then $[\Delta: B]<\infty$ by Lemma I. 1.1. Also $B$ is a normal subgroup of $\Delta$ with $B^{\prime} \leqq A^{\prime} \leqq Z$ so $B^{\prime}$ is finite. Write

$$
\Delta=\left\langle B, w_{1}, w_{2}, \cdots, w_{t}\right\rangle \text {. }
$$

Then by Lemma I. 1.1,

$$
W=B \cap\left(\bigcap_{1}^{t} \mathbf{C}_{\Delta}\left(w_{i}\right)\right)
$$

has finite index in $\Delta$. Since $B / B^{\prime}$ is abelian it then follows that $W B^{\prime} / B^{\prime}$ is a central subgroup of finite index in $\Delta / B^{\prime}$ and hence by Lemma I. 2.1, $\left(\Delta / B^{\prime}\right)^{\prime}$ is finite. Since $B^{\prime}$ is finite and $\left(\Delta / B^{\prime}\right)^{\prime}=\Delta^{\prime} / B^{\prime}$ we have $\left|\Delta^{\prime}\right|<\infty$ and the theorem is proved.

It is interesting to note that, in contrast to Theorem I. 9.4, the degree of the weak polynomial indentity does not play an important role here. For example, in the proof of (i) $\Rightarrow$ (ii) above a weak polynomial was obtained whose degree was independent of $\left|\Delta^{\prime}\right|$. Thus $\left|\Delta^{\prime}\right|$ cannot be bounded by a function of the degree. The same is true of the index $[G: \Delta]$ as the following example indicates.

Suppose that $G=\Delta H$ where $\Delta$ is abelian, $H$ is finite and $|H| \neq 0$ in $K$. For example, we could take $G=Z \nmid Z_{n}$ where $Z$ is infinite cyclic and $Z_{n}$ is cyclic of order $n$. Set

$$
e=\frac{1}{|H|} \sum_{x \in H} x
$$

so that $e$ is an idempotent in $K[G]$. If $h \in H$ then $h e=e$ and thus $e K[G] e$ is spanned by the terms eye with $y \in \Delta$. Now

$$
\begin{aligned}
\text { eye } & =\frac{1}{|H|} e \sum_{x \in H} y x=\frac{1}{|H|} e \sum_{x \in H} x y^{x} \\
& =\frac{1}{|H|} e \sum_{x \in H} y^{x} .
\end{aligned}
$$

Since $\Delta$ is abelian and $G=\Delta H$ it follows easily that $\Sigma_{x} y^{x}$ is central in $K[G]$. This shows that $e K[G] e$ is commutative and hence satisfies a polynomial identity of degree 2. Thus $K[G]$ satisfies a nondegenerate weak polynomial identity of degree 2 independent of the index $[G: \Delta]$.

5. Miscellany. Let $P$ be a nonabelian $p$-group of order $p^{3}$. If $K$ is a field of characteristic $p$ then it is easy to see that $K[P]$ is Lie nilpotent of class $p$. Thus $K[P]$ satisfies a polynomial identity of degree $p+1$. Now it would be interesting to find the minimal degree 
of the polynomial identities satisfied by $K[P]$ and the following a mild step in that direction.

THEOREM 5.1. Let $K$ be a field and let $P$ be a finite nonabelian p-group. If $K[P]$ satisfies a polynomial of degree $n$, then $n>\sqrt[3]{6 p}$.

Proof. Since $P^{\prime} \neq\langle 1\rangle$ we can choose $N$ normal in $P$ with $\left[P^{\prime}: N\right]=p$. If $\bar{P}=P / N$, then $K[\bar{P}]$ also satisfies a polynomial identity of degree $n$ and $\bar{P}^{\prime}$ is central of order $p$. Thus it suffices to assume that $P=\bar{P}$ or equivalently that $P^{\prime}$ is central of order $p$. Choose $x, y \in P$ which do not commute and let $z=x y x^{-1} y^{-1} \neq 1$. Then $P^{\prime}=\langle z\rangle$ and $z$ is central of order $p$. We have $x y=z y x$ and hence by induction $x y^{i}=z^{i} y^{i} x$.

We may assume by Lemma I. 5.3 that $K[P]$ satisfies the multilinear identity

$$
f\left(\zeta_{1}, \zeta_{2}, \cdots, \zeta_{n}\right)=\rho_{1} \cdots \rho_{2} \rho_{n}+\sum_{\sigma \neq 1} a_{o} \zeta_{\sigma(1)} \zeta_{\sigma(2)} \cdots \zeta_{\sigma(n)}
$$

and we set $\zeta_{i}=x y^{i}$. Consider the $\sigma$ monomial evaluated here. We have

$$
\mu_{\sigma}=x y^{\sigma(1)} x y^{\sigma(2)} \cdots x y^{\sigma(n)} .
$$

Now shift all the $y$ 's to the left starting with $y^{\sigma(1)}$. Since $y^{o(i)}$ must pass precisely $i x$ 's we have, using $x y^{j}=z^{j} y^{j} x$

$$
\mu_{\sigma}=z^{\Sigma_{1}^{n}{ }^{i \sigma(i)}} y^{\Sigma_{1}^{n} \sigma(i)} x^{n}=z^{\Sigma_{1}^{n}{ }^{i \sigma(i)}} y^{n(n+1) / 2} x^{n} .
$$

For each $\sigma$ we define the integral vector $v_{\sigma}$ by

$$
v_{\sigma}=\langle\langle\sigma(1), \sigma(2), \cdots, \sigma(n)\rangle\rangle
$$

so that $v_{1}=\langle\langle 1,2, \cdots, n\rangle\rangle$ and

$$
\mu_{\sigma}=z^{v_{1} \cdot v_{\sigma}} y^{n(n+1) / 2} x^{n}
$$

where $v_{1} \cdot v_{\sigma}$ is the usual dot product of vectors.

We consider the values $v_{1} \cdot v_{\sigma}$. By the Cauchy-Schwarz inequality

$$
\left(v_{1} \cdot v_{\sigma}\right)^{2} \leqq\left(v_{1} \cdot v_{1}\right)\left(v_{\sigma} \cdot v_{\sigma}\right)
$$

with equality if and only if $v_{\sigma}$ is a scalar times $v_{1}$ and hence in our case if and only if $\sigma=1$. Since clearly $v_{\sigma} \cdot v_{\sigma}=v_{1} \cdot v_{1}$ we have for all $\sigma \neq 1,\left(v_{1} \cdot v_{\sigma}\right)^{2}<\left(v_{1} \cdot v_{1}\right)^{2}$ so

$$
v_{1} \cdot v_{o}<v_{1} \cdot v_{1}=\sum_{1}^{n} i^{2}=n(n+1)(2 n+1) / 6
$$

since these are all positive quantities.

Clearly zero is a lower bound for all $v_{1} \cdot v_{\sigma}$. A better bound is 
obtained as follows. For each $\sigma$ define $\sigma^{\prime}$ by $\sigma^{\prime}(i)=n+1-\sigma(i)$. Then $\sigma^{\prime}$ is clearly also a permutation and $v_{\sigma^{\prime}}=v-v_{\sigma}$ where

$$
v=\langle\langle n+1, n+1, \cdots, n+1\rangle\rangle \text {. }
$$

By the above

$$
v_{1} \cdot v_{1} \geqq v_{1} \cdot v_{\sigma^{\prime}}=v_{1} \cdot\left(v-v_{\sigma}\right)=v_{1} \cdot v-v_{1} \cdot v_{\sigma}
$$

and thus

$$
v_{1} \cdot v_{\sigma} \geqq v_{1} \cdot v-v_{1} \cdot v_{1}=(n+1) \sum_{1}^{n} i-\sum_{1}^{n} i^{2}=n(n+1)(n+2) / 6 .
$$

Returning to the polynomial identity we have

$$
0=f\left(x y^{1}, x y^{2}, \cdots, x y^{n}\right)=\left\{z^{v_{1} \cdot v_{1}}+\sum_{\sigma \neq 1} a_{\sigma} z^{v_{1} \cdot v_{\sigma}}\right\} y^{n\langle n+1) / 2} x^{n} .
$$

It therefore follows that the $z^{v_{1} \cdot v_{1}}$ terms must be cancelled by other terms in the sum and thus for some $\sigma \neq 1$ we have $z^{v_{1} \cdot v_{1}}=z^{v_{1} \cdot v_{\sigma}}$. Hence $p \mid\left(v_{1} \cdot v_{1}-v_{1} \cdot v_{\sigma}\right)$. Now by the above

$$
\begin{aligned}
0<v_{1} \cdot v_{1}-v_{1} \cdot v_{\sigma} & \leqq n(n+1)(2 n+1) / 6-n(n+1)(n+2) / 6 \\
& =n(n-1)(n+1) / 6
\end{aligned}
$$

and since $p$ divides $v_{1} \cdot v_{1}-v_{1} \cdot v_{\sigma}$ we must have $n(n-1)(n+1) / 6 \geqq p$. Thus $n^{3}>n\left(n^{2}-1\right) \geqq 6 p$ and $n>\sqrt[3]{6 p}$. This completes the proof.

We will need the following few facts on locally finite groups.

LEMma 5.2. Let $G$ be an arbitrary group.

(i) Let $N$ be a normal subgroup of $G$ and suppose that both $N$ and $G / N$ are locally finite. Then $G$ is locally finite.

(ii) Lot $M$ be the subgroup of $G$ generated by all locally finite normal subgroups of $G$. Then $M$ is locally finite and $G / M$ has no nonidentity finite normal subgroup.

Proof. (i) Let $H$ be a finitely generated subgroup of $G$. Then $H N / N$ is a finitely generated subgroup of $G / N$ and thus

$$
H N / N \cong H /(H \cap N)
$$

is finite. Since $H$ is finitely generated and $[H: H \cap N]<\infty$ we see that $H \cap N$ is finitely generated by Lemma I. 7.1. Thus $H \cap N$ is finite and this result follows.

(ii) Suppose $N_{1}$ and $N_{2}$ are locally finite normal subgroups of $G$. Then $N_{1} N_{2}$ is normal in $G$ and $N_{1} N_{2} / N_{1} \cong N_{2} /\left(N_{1} \cap N_{2}\right)$ is clearly a locally finite group. Thus by (i) above $N_{1} N_{2}$ is locally finite. By induction we 
see that any finite number of locally finite normal subgroups of $G$ generate a locally finite group. Since any finitely generated subgroup of $M$ is contained in the group generated by a finite number of locally finite normal subgroups we see that $M$ is locally finite. Let $H / M$ be a normal subgroup of finite order in $G / M$. Then $H$ is normal in $G$ and $H$ is locally finite by (i) so $H=M$ and $H / M=\langle 1\rangle$.

There are a number of interesting characteristic subgroups of $G$ which are related to the question of $K[G]$ being prime or semiprime.

Let $\mathscr{S}$ be the set of normal subgroups $N$ of $G$ such that $K[G / N]$ is prime. By Theorem I. 2.5 this condition is independent of the field $K$ and in fact $N \in \mathscr{S}$ if and only if $\Delta(G / N)$ is torsion free abelian. Set

$$
\Omega=\Omega(G)=\bigcap_{N \in S} N .
$$

Then $\Omega$ is clearly a characteristic subgroup of $G$.

Let $p$ be a prime and let $\mathscr{S}_{p}$ be the set of normal subgroups $N$ of $G$ such that $K[G / N]$ is semiprime for some field $K$ of characteristic p. By Theorem I. 8.6 this condition is independent of the field $K$ and in fact $N \in \mathscr{S}_{p}$ if and only if $\Delta(G / N)$ has no elements of order $p$. Set

$$
\Omega_{p}=\Omega_{p}(G)=\bigcap_{N \in p_{p}} N .
$$

Then $\Omega_{p}$ is clearly a characteristic subgroup of $G$.

Let $\mathbf{O}^{p \prime}(G)$ denote the subgroup of $G$ generated by all elements of order a power of $p$.

Theorem 5.3. With the above notation we have

(i) $K[G / \Omega]$ is prime for any field $K$ and hence $\Omega$ is the unique minimal member of $\mathscr{S}$.

(ii) $K\left[G / \Omega_{p}\right]$ is semiprime for any field $K$ of characteristic $p$ and hence $\Omega_{p}$ is the unique minimal member of $\mathscr{S}_{p}$.

(iii) $\Omega$ is a characteristic locally finite subgroup of $G$.

(iv) $\Omega_{p}$ is a characteristic locally finite subgroup of $G$. Furthermore $\Omega_{p}=\mathbf{0}^{p \prime}\left(\Omega_{p}\right) \subseteq \mathbf{0}^{p \prime}(\Omega)$.

Proof. We start with a simple observation. Let $N \supseteqq H$ be normal subgroups of $G$. Let $\bar{x}=x H$ be an element of order $k$ in $\Delta(G / H)$. Then $x^{k} \in H \cong N$ and all conjugates of $x$ in $G$ are contained in finitely many cosets of $H$ and hence of $N$. Thus $\widetilde{x}=x N \in \Delta(G / N)$ and $\widetilde{x}^{k}=1$ so the order of $\widetilde{x}$ divides $k$.

(i) Let $\bar{x}=x \Omega$ be an element of finite order in $\Delta(G / \Omega)$ and let $N \in \mathscr{S}$. Then by the above $\widetilde{x}=x N$ is an element of finite order in $\Delta(G / N)$ so $\widetilde{x}=1$ by Theorem I. 2.5. Thus $x \in \bigcap N=\Omega$ and $\bar{x}=1$. 
Hence $K[G / \Omega]$ is prime by Lemma I. 2.2 and Theorem I. 2.5.

(ii) The proof here is the same as (i) except that we let $\bar{x}$ have order a power of $p$ and we apply Theorem I. 3.1.

(iii) Let $M$ be the subgroup of $G$ given in Lemma 5.2. ii. Then $G / M$ has no nonidentity finite normal subgroups so by Theorem I. 2.5., $M \in \mathscr{S}$. Since $\Omega \subseteq M$ and $M$ is locally finite, this result follows.

(iv) Since any prime ring is also semiprime we have $\mathscr{S} \subseteq \mathscr{S}_{p}$ and hence $\Omega_{p} \subseteq \Omega$. Thus by (iii) $\Omega_{p}$ is locally finite. Let $N=\mathbf{0}^{p \prime}\left(\Omega_{p}\right)$ so that $N$ is also normal in $G$. Since $\Omega_{p}$ is periodic we see that $\Omega_{p} / N$ has no elements of order $p$. Let $x N$ be an element of order $p$ in $\Delta(G / N)$. Then $x \Omega_{\rho}$ is an element of order $p$ or 1 in $\Delta\left[G / \Omega_{p}\right]$. Since $K\left[G / \Omega_{p}\right]$ is semi-prime for any field $K$ of characteristic $p$ by (ii), Theorem I. 3.6 implies that $x \Omega_{p}$ has order 1 so $x \in \Omega_{p}$. Since $x^{p} \in N$ and $\Omega_{p} / N$ has no elements of order $p$ we conclude that $x \in N$ and $x N$ has order 1 , a contradiction. Thus $\Delta(G / N)$ has no elements of order $p$ and $N \in \mathscr{S}_{p}$ by Theorem I. 3.6. Hence $\Omega_{p} \subseteq N$ so $\Omega_{p}=N=\mathbf{0}^{p \prime}\left(\Omega_{p}\right)$. Finally $\Omega_{p} \leqq \Omega$ implies $\mathbf{0}^{p^{\prime}}\left(\Omega_{p}\right) \leqq \mathbf{0}^{p^{\prime}}(\Omega)$ and the result follows.

It is not necessarily true in (iv) above that $\Omega_{p}=\mathbf{0}^{p \prime}(\Omega)$. Let $q$ be a prime distinct from $p$ and let $A$ be an infinite elementary abelian $q$ group. Set $G=A \otimes_{\sigma} Z_{p}$, the semidirect product of $A$ by $Z_{p}$, the cyclic group of order $p$, where $Z_{p}$ acts on $A$ by permuting a basis in cycles of length $p$. Clearly $A=\Delta(G)$ so by Theorem I. 3.6 we have $\Omega_{p}(G)=\langle 1\rangle$. If $N$ is normal in $G$ with $K[G / N]$ prime then by Theorem I. 2.5 we must have $N \supseteqq A$. Then $G / N$ is finite so $G=N$. This shows that $\Omega(G)=G$ and hence $\mathbf{O}^{p \prime}(\Omega) \neq \Omega_{p}$ in this case.

6. Solvable subgroups. Probably the chief stumbling block in obtaining characteristic $p$ analogues of Theorem 5.3 of [7] has been the use of Jordan's theorem on complex linear groups in its proof. In this section we offer a simple argument which can be used instead of it in characteristic 0 . In addition we announce an appropriate analogue in characteristic $p$ and we indicate some interesting consequences. Proofs of the latter results require a complete reformulation of the pertinent parts of [7] from a character-theoretic to a moduletheoretic point of view and they will appear elsewhere.

Lemma 6.1. Let $G$ be a finite group with r.b.n. (in the notation of [7]). If $G \neq\langle 1\rangle$ then there exists an element $x \in G, x \neq 1$ with $[G: \mathbf{C}(x)] \leqq n^{2}$.

Proof. Say $G$ has $m$ irreducible characters and hence also $m$ conjugacy classes. Since $G \neq\langle 1\rangle$ we have $m>1$. Let the degrees of the characters be $1=n_{1}, n_{2}, \cdots, n_{m}$ where $n_{1}$ corresponds to the 
principal character. Then

$$
|G|=n_{1}^{2}+n_{2}^{2}+\cdots+n_{m}^{2} \leqq 1+(m-1) n^{2}
$$

since $G$ has r.b.n.. Let the conjugacy classes of $G$ have sizes $1=c_{1}, c_{2}, \cdots, c_{m}$ where $c_{1}$ corresponds to the identity class. If $c=\min _{i \neq 1} c_{i}$ then

$$
|G|=c_{1}+c_{2}+\cdots+c_{m} \geqq 1+(m-1) c .
$$

Taken together these two inequalities then yield $n^{2} \geqq c=[G: \mathbf{C}(x)]$ for some $x \neq 1$.

Lemma 6.2. Let $G$ be a finite group with r.b.n. If $G \neq\langle 1\rangle$ then $G$ has a normal subgroup $N$ with $[G: N] \leqq\left(n^{2}\right) !$ and $\mathbf{C}(N) \neq\langle 1\rangle$.

Proof. Let $x$ be as in Lemma 6.1 and let $N$ be the core of $\mathrm{C}(x)$, that is, $N$ is the intersection of all conjugates of $\mathrm{C}(x)$. Then $[G: N] \leqq\left(n^{2}\right)$ ! since $[G: \mathbf{C}(x)] \leqq n^{2}$ and $x \in \mathbf{C}(N)$ so $\mathbf{C}(N) \neq\langle 1\rangle$.

Jordan's theorem is used on page 896 of [7] to bound the order of a group $E$ satisfying

(1) $E$ is nonabelian.

(2) All proper homomorphic images of $E$ are abelian.

(3) $E$ has no nonidentity normal abelian subgroup. We use Lemma 6.2 instead as follows.

LEMMA 6.3. Let $E$ be a finite group with r.b.n. and let $E$ satisfy (1), (2) and (3) above. Then $|E| \leqq\left(n^{2}\right)$ !

Proof. Let $N$ be the normal subgroup of $E$ given by Lemma 6.2. Suppose $N \neq\langle 1\rangle$. Then by (2), $N \supseteqq E^{\prime}$. Since $\mathrm{C}(N)$ is normal in $E$ and $\mathbf{C}(N) \neq\langle 1\rangle$ we have also $\mathbf{C}(N) \supseteqq E^{\prime}$. Thus $N \cap \mathbf{C}(N)=$ $\mathbf{Z}(N) \supseteqq E^{\prime}$ and $E^{\prime}$ is abelian, contradicting (1) and (3). Hence $N=\langle 1\rangle$ and $|E|=[E: N] \leqq\left(n^{2}\right)$ !

The appropriate characteristic $p$ analogue of bounded representation is of course that the group ring satisfy a polynomial identity. Using a combinatorial argument similar to that of Theorem I. 6.2 we can obtain

LEMma 6.4. Let $G$ be finite and nonabelian and let $K$ be any field. If $K[G]$ satisfies a polynomial identity of degree $n$ then there exists an element $x \in G-\mathbf{Z}(G)$ with $[G: \mathbf{C}(x)] \leqq(n !)^{2}$. 
Of course this immediately yields analogues of Lemmas 6.2 and 6.3. Define the integer valued function $\mathfrak{Y}(n)$ as follows.

$$
\mathfrak{Q}(n)=(n !)^{n \cdot(n !)^{2}} \text {. }
$$

Then using the above argument instead of Jordan's theorem we obtain the following replacements for Theorem I. 8.2 and I. 9.4 respectively.

THEOREM 6.5. Let $G$ be a finite group and let $K$ be a field with $\left|G^{\prime}\right| \neq 0$ in $K$. If $K[G]$ satisfies a polynomial identity of degree $n$, then $G$ has an abelian subgroup $A$ with $[G: A] \leqq \mathfrak{X}(n)$.

THEOREM 6.6. Let $K[G]$ be a semiprime group ring which satisfies a polynomial identity of degree $n$. Then $G$ has an abelian subgroup $A$ with $[G: A] \leqq n ! \mathfrak{U}(n)$.

If $K[G]$ is not semiprime we can still obtain results of interest. Let $G$ be an arbitrary group and let $\mathrm{S}(G)$ be the subgroup of $G$ generated by all normal solvable subgroups of $G$. It follows easily that $\mathbf{S}(G)$ is a characteristic locally solvable subgroup of $G$. In particular, if $G$ is finite, then $\mathbf{S}(G)$ is the unique maximal normal solvable subgroup of $G$.

THEOREM 6.7. Let $G$ be a finitely generated group and let $K$ be any field. If $K[G]$ satisfies a polynomial identity of degree $n$ then $\mathbf{S}(G)$ is solvable and $[G: \mathbf{S}(G)] \leqq \mathfrak{U}(n)$.

THEOREM 6.8. Let $G$ be an arbitrary group and let $K$ be any field. If $K[G]$ satisfies a polynomial identity of degree $n$ then $[G: \mathbf{S}(G)] \leqq n ! \cdot \mathfrak{Y}(n)$.

\section{REFERENCES}

I. D. S. Passman, Linear identities in group rings, Pacific J. Math., 36 (1971).

13. S. A. Amitsur, Generalized polynomial identities and pivotal monomials, Trans. A.M.S., 114 (1965), 210-226.

14. I. N. Herstein, Noncommutative Rings, Carus Mathematical Monographs, No. 15, M. A. A., 1968.

Received May 25, 1970.

Institute for Defense Analyses

Princeton, New Jersey

UNIVERSITY OF WISCONSIN 



\section{PACIFIC JOURNAL OF MATHEMATICS}

\section{EDITORS}

H. SAMELson

Stanford University

Stanford, California 94305

R. R. PHelPS

University of Washington

Seattle, Washington 98105
J. Dugundu

Department of Mathematics

University of Southern Californle

Los Angeles, California 9.0007

RICHARD ARENS

University of California

Los Angeles, California 9.0024

\section{ASSOCIATE EDITORS}
E. F. BECKENBACH
B. H. NeumanN
F. WOLE
K. Yoshida

\section{SUPPORTING INSTITUTIONS}

UNIVERSITY OF BRITISH COLUMBIA

CALIFORNIA INSTITUTE OF TECHNOLOGY

UNIVERSITY OF CALIFORNIA

MONTANA STATE UNIVERSITY

UNIVERSITY OF NEVADA

NEW MEXICO STATE UNIVERSITY

OREGON STATE UNIVERSITY

UNIVERSITY OF OREGON

OSAKA UNIVERSITY

UNIVERSITY OF SOUTHERN CALIFORNIA

\author{
STANFORD UNIVERSITY \\ UNIVERSITY OF TOKYO \\ UNIVERSITY OF UTAH \\ WASHINGTON STATE UNIVERSITY \\ UNIVERSITY OF WASHINGTON \\ AMERICAN MATHEMATICAL SOCIETY \\ CHEVRON RESEARCH CORPORATION \\ NAVAL WEAPONS CENTER
}

The Supporting Institutions listed above contribute to the cost of publication of this Journal, but they are not owners or publishers and have no responsibility for its content or policies.

Mathematical papers intended for publication in the Pacific Journal of Mathematics should be in typed form or offset-reproduced, (not dittoed), double spaced with large margins. Underline Greek letters in red, German in green, and script in blue. The first paragraph or two must be capable of being used separately as a synopsis of the entire paper. The editorial "we" must not be used in the synopsis, and items of the bibliography should not be cited there unless absolutely necessary, in which case they must be identified by author and Journal, rather than by item number. Manuscripts, in duplicate if possible, may be sent to any one of the four editors. Please classify according to the scheme of Math. Rev. Index to Vol. 39. All other communications to the editors should be addressed to the managing editor, Richard Arens, University of California, Los Angeles, California, 90024.

50 reprints are provided free for each article; additional copies may be obtained at cost in multiples of 50 .

The Pacific Journal of Mathematics is published monthly. Effective with Volume 16 the price per volume (3 numbers) is $\$ 8.00$; single issues, $\$ 3.00$. Special price for current issues to individual faculty members of supporting institutions and to individual members of the American Mathematical Society: $\$ 4.00$ per volume; single issues $\$ 1.50$. Back numbers are available.

Subscriptions, orders for back numbers, and changes of address should be sent to Pacific Journal of Mathematics, 103 Highland Boulevard, Berkeley, California, 94708.

PUBLISHED BY PACIFIC JOURNAL OF MATHEMATICS, A NON-PROFIT CORPORATION

Printed at Kokusai Bunken Insatsusha (International Academic Printing Co., Ltd.), 7-17, Fujimi 2-chome, Chiyoda-ku, Tokyo, Japan. 


\section{Pacific Journal of Mathematics}

\section{Vol. 36, No. 2 December, 1971}

George E. Andrews, On a partition problem of H. L. Alder ............ 279

Thomas Craig Brown, An interesting combinatorial method in the theory of locally finite semigroups .......................... 285

Yuen-Kwok Chan, A constructive proof of Sard's theorem ............. 291

Charles Vernon Coffman, Spectral theory of monotone Hammerstein

operators...................................... 303

Edward Dewey Davis, Regular sequences and minimal bases .......... 323

Israel (Yitzchak) Nathan Herstein and Lance W. Small, Regular elements in

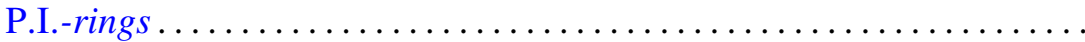

Marcel Herzog, Intersections of nilpotent Hall subgroups ..............

W. N. Hudson, Volterra transformations of the Wiener measure on the space

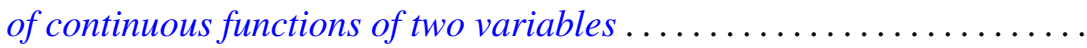

J. H. V. Hunt, An n-arc theorem for Peano spaces ................ 351

Arnold Joseph Insel, A decomposition theorem for topological group

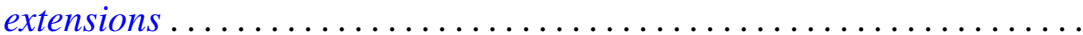

Caulton Lee Irwin, Inverting operators for singular boundary value

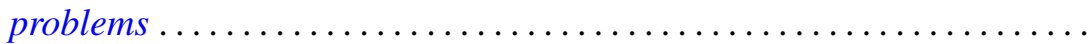

Abraham A. Klein, Matrix rings of finite degree of nilpotency ............ 387

Wei-Eihn Kuan, On the hyperplane section through a rational point of an algebraic variety...

John Hathway Lindsey, II, On a six-dimensional projective representation of $\mathrm{PSU}_{4}(3)$

Jorge Martinez, Approximation by archimedean lattice cones ...

J. F. McClendon, On stable fiber space obstructions .........

Mitsuru Nakai and Leo Sario, Behavior of Green lines at the Kuramochi boundary of a Riemann surface ....................

Donald Steven Passman, Linear identities in group rings. I. .

Donald Steven Passman, Linear identities in group rings. II ...

David S. Promislow, The Kakutani theorem for tensor products of

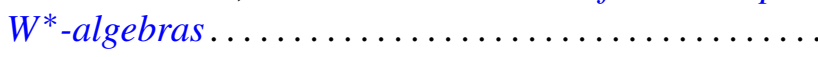

Richard Lewis Roth, On the conjugating representation of a finite group

Bert Alan Taylor, On weighted polynomial approximation of entire functions...

William Charles Waterhouse, Divisor classes in pseudo Galois

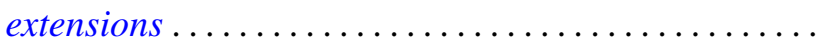

Chi Song Wong, Subadditive functions ...

Ta-Sun $\mathrm{Wu}$, A note on the minimality of certain bitransformation groups 